P391 A RARE ASSOCIATION IN THE $21^{\text {ST }}$ CENTURY: SHIGELLA FLEXNERI ENTEROCOLITIS AND SCABIES IN A SEVERELY MALNOURISHED INFANT

${ }^{1}$ Alexandra Mititelu*, ${ }^{2}$ Serban Tomescu, ${ }^{1}$ Alexandra Micu Chis, ${ }^{1}$ Mihaela Rednic, ${ }^{1,3}$ Kinga Cristina Slavescu. ${ }^{1}$ Clinical Emergency Hospital for Children, Cluj-Napoca, Romania; ${ }^{2}$ Clinical Hospital of Infectious Diseases, Cluj-Napoca, Romania; ${ }^{3}$ University of Medicine and Pharmacy 'Iuliu Hatieganu', Cluj-Napoca, Romania

\subsection{6/archdischild-2019-epa.737}

Background Shigellosis (previously bacillary dysentery) was the primary diarrhoeal disease of World War 1 but continues to be an important public health problem in developing and undeveloped countries. The disease is difficult to prevent, because only a small number of bacteria can cause illness and can lead to various extra-intestinal complications like septicemia. On the other hand, scabies is a contagious skin infection caused by female Sarcoptes scabies. The purpose of this clinical case report is to highlight the unusual association of two concomitant unlikely infection in a developed country, in a malnourished infant and a possible diagnostic pitfall.

Case presentation We report the case of a 10 -weeks-old premature infant brought in for multiple episodes of watery diarrhoea, fever and multiple disseminated cutaneous papulous lesions. At that time the infant was severely malnourished. Based on the clinical and laboratory findings a diagnostic of bacterial enterocolitis, hypoproteinemia, hypoalbuminemia, hyponatremia and severe anemia, complicated with septicemia was made. Because of the excoriated papules and small nodules and family history (both siblings presented the same lesions) a high suspicion of scabies was made.We initiated treatment with intravenous antibiotics (Cefotaxime, Ampicillin and Gentamicin), iv fluids, antidiarrheals, albumin and blood transfusion. After the isolation of Shigella flexneri from the stool culture, the patient was transferred in the Infectious Disease Department, where he received Ceftriaxone, iv fluids and Benzyl Benzoate for the cutaneous lesions. The patient's evolution was favourable with the partial remission of the diarrhoeal stools, cutaneous lesions and normalised laboratory tests. Due to the severe malnutrition the infant was afterwards transferred to the Dystrophic Department, where they suspected further cow's milk protein allergy and secondary lactase deficiency (because of the persistence of some cutaneous lesions and modified stools with negative stool culture). After passing to a lactose free and extensive hydrolysed protein formula all symptoms disappearedand the infant gain weight.

Discussion This case illustrates the unlikely association of two rare infections in the 21 st century, in a developed country, in a malnourished infant and the overlap of symptoms between these infections and the cow's milk protein allergy.

\section{P392 INFLUENZA IN CHILDREN: CAN WE PREDICT OUTCOMES?}

\footnotetext{
${ }^{1}$ Jennifer Cox* ${ }^{1}$ Laura Whitla, ${ }^{1}$ Elizabeth Murphy, ${ }^{1,2}$ Montaseur Nadeem, ${ }^{1,2}$ David Coghlan,

1,2Basil Elnazir. 'Tallaght University Hospital, Dublin, Ireland; ${ }^{2}$ Trinity College Dublin, Dublin, Ireland
}

10.1136/archdischild-2019-epa.738
Aims To examine whether there is link between influenza strains in children and length of hospital stay (LOS), transfer to ICU, mortality, secondary bacterial infection (SBI) or peripheral blood tests.

Methods We studied 141 patients attended our hospital between November 2017 and March 2018.

Results Influenza A was reported in 80 (56.7\%), influenza B in $60(42.6 \%)$ and both strains in one. Mean (SD) age was 5.12 (4.30) years. Statistically, patients were divided into two groups (influenza A and influenza B). Admission to paediatric ward was required in 91 patients $(64.54 \%)$, of whom two required transfer to ICU, with no mortality. Mean (SD) LOS was 2.33 (1.92) days, with no link between LOS and influenza viral strains ( $\mathrm{p}$ value 0.35 ).

Of 141 patients, 111 underwent blood tests. The majority had normal total WBC [72 patients (64.9\%)] and neutrophil values [80 (72.1\%)], normal lymphocyte [61 patients $(55.0 \%)]$ and monocyte values [64 (57.7\%)]. However, total WBC, neutrophil, lymphocyte and monocyte values were elevated in 9 (8.1\%), 19 (17.1\%), two (1.8\%) and 47 (42.3\%) patients, respectively. Total WBC, neutrophil, lymphocyte or monocyte values did not differ between the groups ( $\mathrm{p}$ value $0.48,0.52$, 0.71 and 0.38 , respectively).

In terms of predicting influenza strains, neutrophil lymphocyte ratio (NLR) and monocyte lymphocyte ratio (MLR) have low sensitivity, with area under the ROC curve 0.42 and 0.53 , respectively. Mean (SD) C-reactive protein (CRP) value was $14.45(10.12) \mathrm{mg} / \mathrm{L}$, with no difference between the groups ( $p$ value 0.52 ). No organisms were detected in 79 patients who underwent peripheral blood culture.

Conclusion Influenza can occur with normal WBC and CRP values. NLR or MLR has no role in predicting influenza serotypes. In this group of patients, the vast majority did not require transfer to ICU. Moreover, there is no link between influenza viral strains and LOS, mortality or SBI.

\section{P393 PERTUSSIS INFECTION; RECENT CONCERNS AND NEW RECOMMENDATIONS}

Ali Raba*, Ibraheem Krebit. Paediatric Emergency Department, Tallaght University Hospital, Dublin, Ireland

\subsection{6/archdischild-2019-epa.739}

Pertussis is a highly contagious bacterial disease which can affect people at any age, and which is significantly underdiagnosed. Its epidemiology has recently changed with a shift in distribution from infants and young children towards older age groups. Immunity from vaccination and from illness wanes over time, leaving adolescents and adults susceptible. These are the main source of infection in infants, in whom significant complications are most common. In order to improve individual and herd immunity and thus reduce transmission of infection to young infants, an adolescent pertussis booster vaccine is recommended. This review discusses recent findings in $B$. pertussis epidemiology and recent advances in the diagnosis, treatment and prevention, and vaccination recommendations for the ultimate elimination of pertussis disease. 